\title{
Hybrid Adaptive Neural Network AUV Controller Design with Sliding Mode Robust Term
}

\author{
Behdad Geranmehr ${ }^{1 *}$, Kamran Vafaee ${ }^{2}$ \\ 1,2 Young Researchers and Elite Club, BuinZahra Branch, Islamic Azad University, BuinZahra, Iran; \\ Behdad.Geranmehr@gmail.com
}

\section{ARTICLE INFO}

Article History:

Received: 26 Oct. 2016

Accepted: 15 Mar. 2017

\section{Keywords:}

AUV

REMUS

RBF NN

SMC

Adaptive

\begin{abstract}
This work addresses an autonomous underwater vehicle (AUV) for applying nonlinear control which is capable of disturbance rejection via intelligent estimation of uncertainties. Adaptive radial basis function neural network (RBF NN) controller is proposed to approximate unknown nonlinear dynamics. The problem of designing an adaptive RBF NN controller was augmented with sliding mode robust term to improve trajectory tracking and regulation in presence of uncertainties. Moreover, stability proof of proposed control scheme was shown with Lyapunov theory. Furthermore, the control, design and simulation results are provided without any simplification of the entire system. Although the design approach of this paper is implemented on REMUS this point of view can be applied on any AUV using the same technique.
\end{abstract}

\section{Introduction}

Nowadays, in addition to pervasive AUVs applications in a verity of industries such as scientific, commercial and military, AUVs are taken into consideration for hard nonlinearity, uncertainty and unknown parameters in their dynamic modeling. Furthermore, the complicated behavior of AUVs and limitations on designing controllers for six-DOF models of underwater vehicles leads to some simplifications such as linearizing and decoupling to overcome complexity. In the scope of this paper, steering and diving control of an AUV are considered for regulation and tracking problems.

An adaptive sliding mode heading control approach of AUV is proposed by Chu and Zhu [1]. Geranmehr and Rafee Nekoo presented a study on the fully coupled control of AUVs in six degrees of freedom (DOF) using the SDRE control approach [2], they also investigated on SDRE and robust term for diving and steering mode [3, 4]. Other research applied the sliding mode control and the backstepping technique for controlling underwater vehicles [5] and for spacecraft attitude control [6]. At first, Lewis and et al. developed adaptive RBF NN controller for seriallink robot arm with sliding mode robust term to guarantee tracking performance [7]. Recently, a radial basis function neural network adaptive controller is applied in different applications [8-11].

The main contribution of this work is: the control problem of REMUS in position and attitude control for steering and diving autopilot modes are investigated via adaptive radial based function neural networks. In addition, underwater ocean current is added to simulate practical environment and highlight the impact of adaptive and robust term in control design.

The remainder of the article is presented as follows: a mathematical model of the AUV for steering and diving is expressed in Section 2; structure of the controller is presented in Section 3; implementation control law for REMUS is presented in Section 4 and the results of simulation are provided in Section 5; finally, conclusions are expressed in Section 6.

\section{Mathematical Model of AUV}

Due to the fact that hydrodynamic drag, damping and lift forces, Coriolis and centripetal forces, gravity, buoyancy forces and thrust the system dynamics of AUVs are highly nonlinear, coupled and time varying [12]. A schematic view of an AUV with related coordinate systems is presented in Fig. 1 to show a six-DOF AUV model. As demonstrated, special reference frames were established to describe motion of the AUV and to set up a six-DOF nonlinear mathematical model. The two reference frames applied to the model were those of a fixed reference frame (earth or inertial coordinate system) and a motion reference frame (body-fixed coordinate system). 


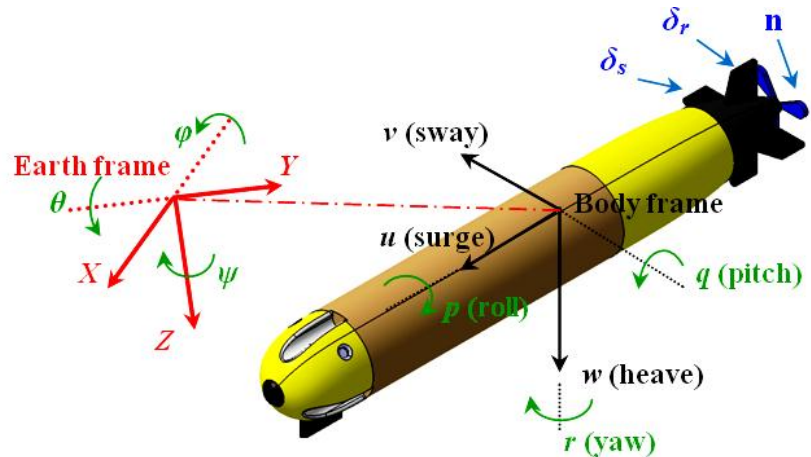

Figure 1. The schematic of the six-DOF model of AUV: REMUS.

Based on this notation, the general motion of a marine vehicle in six-DOF can be described by $\boldsymbol{\eta}=\left[\begin{array}{ll}\boldsymbol{\eta}_{1} & \boldsymbol{\eta}_{2}\end{array}\right]^{T}=\left[\begin{array}{llllll}x_{c} & y_{c} & z_{c} & \phi & \theta & \psi\end{array}\right]^{T}$ and $\mathbf{v}=\left[\begin{array}{ll}\mathbf{v}_{1} & \mathbf{v}_{2}\end{array}\right]^{T}=\left[\begin{array}{llllllll}u & v & w & p & q & r\end{array}\right]^{T}$. A Aeneral description of six-DOF nonlinear equation of AUV motion is introduced as follows $[2,12]$ :

$\left\{\begin{array}{l}\dot{\boldsymbol{\eta}}=\mathbf{J}(\boldsymbol{\eta}) \mathbf{v} \\ \mathbf{M}(\boldsymbol{\eta}) \dot{\boldsymbol{v}}+\mathbf{C}(\boldsymbol{\eta}, \mathbf{v}) \mathbf{v}+\mathbf{D}(\boldsymbol{\eta} \mathbf{v}) \mathbf{v}+\mathbf{G}(\boldsymbol{\eta})=\tau\end{array}\right.$

where $\mathbf{M} \in \mathfrak{R}^{6 \times 6}$ is the inertial matrix, $\mathbf{C}(\mathbf{v}) \in \mathfrak{R}^{6 \times 6}$ is the matrix of Coriolis and centripetal terms, $\mathbf{D}(\boldsymbol{v}) \in \Re^{6 \times 6}$ is damping matrix, $\mathbf{G}(\boldsymbol{\eta}) \in \mathfrak{R}^{6 \times 1}$ is the vector of gravitational/buoyancy forces and moments, $\tau \in \mathfrak{R}^{6 \times 1}$ is the vector of control inputs relating to the forces and moments acting on the AUV.

A global frame of reference of the kinematics of the AUV model is defined using Euler angles. The kinematics equation is then expressed as:

$$
\dot{\eta}=J(\eta) v=\left[\begin{array}{cc}
R(\eta) & 0_{3 \times 3} \\
\mathbf{0}_{3 \times 3} & T(\eta)
\end{array}\right]\left[\begin{array}{c}
v_{1} \\
v_{2}
\end{array}\right],
$$

where, $\mathbf{R}(\boldsymbol{\eta})$ is the rotation matrix from the body frame to the inertial frame, and $\mathbf{T}(\boldsymbol{\eta})$ is the angular velocity transformation from the body frame to the inertial frame. The state vector is considered as $\mathbf{x}=\left[\begin{array}{llllllllllll}x_{c} & y_{c} & z_{c} & \varphi & \theta & \psi & u & v & w & p & q & r\end{array}\right]^{T}$ and the state-space representation of the system is written in the following form:

$$
\dot{\mathbf{x}}(t)=\mathbf{f}(\mathbf{x}(t))+\mathbf{g}(\mathbf{x}(t)) \mathbf{u}(t),
$$

in which

$$
\begin{aligned}
\mathbf{f}(\mathbf{x}(t)) & =\left[\begin{array}{c}
\mathbf{J}(\boldsymbol{\eta}) \mathbf{v} \\
-\mathbf{M}(\boldsymbol{\eta})^{-1}(\mathbf{C}(\boldsymbol{\eta}, \mathbf{v}) \mathbf{v}+\mathbf{D}(\boldsymbol{\eta}, \mathbf{v}) \mathbf{v}+\mathbf{G}(\boldsymbol{\eta}))
\end{array}\right], \\
\mathbf{g}(\mathbf{x}(t)) & =\left[\begin{array}{c}
\mathbf{0}_{6 \times 1} \\
\mathbf{M}(\boldsymbol{\eta})^{-1}
\end{array}\right] .
\end{aligned}
$$

Comparisons between a coupled system and an uncoupled system have demonstrated that we be able to reduce the complexity of control design via decoupling. The general six-DOF AUV can be categorized according to lightly interacting or noninteracting subsystems which can be described by three uncoupled basic subsystems: speed, heading and depth, [2, 12], as indicated in Table 1.

Table 1. Decoupled subsystems of an underwater vehicle

\begin{tabular}{ccc}
\hline Subsystem & Description & Control inputs \\
\hline Speed & $\mathrm{u}(\mathrm{t})$ & $\mathrm{n}(\mathrm{t})$ \\
Steering & $\mathrm{v}(\mathrm{t}), \mathrm{r}(\mathrm{t}), \psi(\mathrm{t})$ & $\delta \mathrm{r}(\mathrm{t})$ \\
Diving & $\mathrm{w}(\mathrm{t}), \mathrm{q}(\mathrm{t}), \theta(\mathrm{t}), \mathrm{z}(\mathrm{t})$ & $\delta \mathrm{s}(\mathrm{t})$ \\
\hline
\end{tabular}

\subsection{Steering}

Usually, the heading subsystem presents the steering motion of the AUV in the $\mathrm{X}-\mathrm{Y}$ plane with $\mathbf{x}(t)=\left[\begin{array}{lll}\psi & v & r\end{array}\right]^{T}$ state vector which the control input commands the deflection of rudder planes $\left(\delta_{r}\right)$, but in this paper, the semi-coupled steering subsystem is presented. The system state vector in this case is considered as $\mathbf{x}(t)=\left[\begin{array}{ll}\boldsymbol{\eta}(t) & \mathbf{v}(t)\end{array}\right]^{T}$ where $\boldsymbol{\eta}(t)=\left[\begin{array}{ll}x & y\end{array}\right]^{T}$ and $\mathbf{v}(t)=\left[\begin{array}{ll}u & v\end{array}\right]^{T}$. The movement of AUV is controlled by vertical rudder $\left(\delta_{r}\right)$ for translational movement control (steering control). Moreover, the surge speed control is managed by the propeller's speed of rotation (n).

Considering the mentioned states, according to the Eq. (1), the dynamics model for semi-coupled steering system is given as:

$$
\begin{aligned}
& \overbrace{\left[\begin{array}{c}
\dot{z} \\
\dot{\theta}
\end{array}\right]}^{\dot{\eta}}=\overbrace{\left[\begin{array}{cc}
\cos (\theta) & 0 \\
0 & 1
\end{array}\right]\left[\begin{array}{c}
w \\
q
\end{array}\right],}^{\mathbf{J}(\boldsymbol{\eta})}, \\
& \overbrace{\left[\begin{array}{cc}
m-X_{\dot{u}} & 0 \\
0 & m-Y_{\dot{v}}
\end{array}\right]}^{\mathbf{M}}\left[\begin{array}{c}
\dot{w} \\
\dot{q}
\end{array}\right]+\overbrace{\left[\begin{array}{cc}
0 & 0 \\
0 & -Y_{u v} u
\end{array}\right]}^{\mathbf{C}(\mathbf{v})}\left[\begin{array}{l}
w \\
q
\end{array}\right] \\
& +\underbrace{\left[\begin{array}{cc}
-X_{u|u|}|u| & 0 \\
0 & -Y_{v|v|}|v|
\end{array}\right]}_{\mathbf{D}(\mathbf{v})}\left[\begin{array}{l}
w \\
q
\end{array}\right]=\tau,
\end{aligned}
$$

where $\left\{X_{\dot{u}}, Y_{\dot{v}}\right\}$ are added mass coefficients, $Y_{u v}$ is Coriolis and centripetal coefficients which can be derived from hydrodynamic derivatives, $\left\{X_{u|u|}, Y_{v|v|}\right\}$ are nonlinear damping vortex shedding coefficients that can be estimated by calculating the hull drag, $Y_{u u \delta_{r}}$ is sway force coefficient for rudder displacement, $\tau_{p}$ is propeller's thrust reduction factor, and $T_{n|n|}$ is thrust coefficient. The parameters are expressed in [14]. 


\subsection{Diving}

The diving subsystem presents depth motion of the AUV in the X-Z plane. The control input commands deflection of stern planes or bow planes $\delta_{s}$. The system state vector in this case is considered as $\mathbf{x}(t)=\left[\begin{array}{ll}\boldsymbol{\eta}(t) & \mathbf{v}(t)\end{array}\right]^{T} \quad$ where $\quad \boldsymbol{\eta}(t)=\left[\begin{array}{ll}z & \theta\end{array}\right]^{T}$ and $\mathbf{v}(t)=\left[\begin{array}{ll}w & q\end{array}\right]^{T}$.

Assuming a constant speed $\mathrm{u}$ for $\mathrm{xc}$ direction and ignoring other states, according to the Eq. (1), the dynamics model for diving is reduced as:

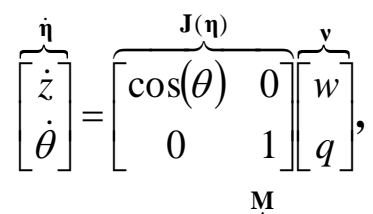

$$
\begin{aligned}
& \overbrace{\left[\begin{array}{cc}
m-Z_{\dot{w}} & -\left(m x_{G}+Z_{\dot{q}}\right) \\
-\left(m x_{G}+M_{\dot{w}}\right) & I_{y}-M_{\dot{w}}
\end{array}\right]}\left[\begin{array}{c}
\dot{w} \\
\dot{q}
\end{array}\right]+ \\
& \overbrace{\left[\begin{array}{cc}
-Z_{u w} u & -Z_{u q} u-m u-m q z_{G} \\
m u-M_{u w} u+m q z_{G} & -M_{u q} u
\end{array}\right]}^{\mathbf{C}(\mathbf{v})}\left[\begin{array}{l}
w \\
q
\end{array}\right]
\end{aligned}
$$$$
+\underbrace{\left[\begin{array}{cc}
-Z_{w|w|}|w| & -Z_{q|q|}|q| \\
-M_{w|w|}|w| & -M_{q|q|}|q|
\end{array}\right]}_{\mathbf{D}(\mathbf{v})}\left[\begin{array}{c}
w \\
q
\end{array}\right]
$$$$
+\underbrace{\left[\begin{array}{c}
\cos (\theta)(B-W) \\
-\sin (\theta)\left(B z_{B}-W z_{G}\right)-\cos (\theta)\left(B x_{B}-W x_{G}\right)
\end{array}\right]}_{\mathbf{G}(\boldsymbol{\eta})}=\tau,
$$

where $I_{y}$ is the moment of inertia about y-axis of the body frame, $\left\{Z_{\dot{w}}, Z_{\dot{q}}, M_{\dot{w}}, M_{\dot{q}}\right\}$ are added mass coefficients, $\left\{Z_{u w}, Z_{u q}, M_{u w}, M_{u q}\right\}$ are Coriolis and centripetal coefficients which can be derived from hydrodynamic derivatives, and $\left\{Z_{w|w|}, M_{q|q|}\right\}$ are nonlinear damping vortex shedding coefficients that can be estimated by calculating the hull drag [14].

\section{Adaptive RBF NN Controller with Sliding Mode Robust Term}

\subsection{RBF Algorithm}

RBF neural network consists of three layers; input layer, hidden layer, and output layer. Radial basis function activates neurons at the hidden layer. The hidden layer has an array of computing units called hidden nodes. Each hidden node contains a center $c$ vector that is a parameter vector of the same dimension as the input vector $x$; the Euclidean distance between the center and the network input vector $x$ is defined by $\left\|x(t)-c_{j}(t)\right\|$. The output of hidden layer can be produced through a nonlinear activation function $h_{j}(t)$ as follows [7]:

$$
h_{j}(t)=\exp \left(-\frac{\left\|x(t)-c_{j}(t)\right\|^{2}}{2 b_{j}^{2}}\right), \quad \mathrm{j}=1, \ldots, \mathrm{m}
$$

where $x$ is input vector, $b_{j}$ notes a positive scalar called a width, $h_{j}$ denotes the output of hidden layer and $m$ notes the number of hidden nodes. The output layer is a linear weighted combination:

$$
y_{i}(t)=\sum_{\mathrm{j}=1}^{\mathrm{m}} w_{j i} h_{j}(t), \quad \mathrm{i}=1, \ldots, \mathrm{n}
$$

where $w$ are the output layer weights, $n$ notes the number of outputs, and $y$ notes the network output.

\subsection{Adaptive RBF Algorithm}

Online adaptive RBF neural network control method is designed based on the Lyapunov stability theory to estimate unknown nonlinear function. As demonstrated in Fig2. We use desired signal $e(t)=\eta_{d}(t)-\eta(t)$ to build input vector as $\left[\begin{array}{ll}e & \dot{e}\end{array}\right]$ and RBF to design $\hat{f}(x)$ for approximating $f(x)$, the output vector is [7]:

$$
\hat{f}(x)=\hat{\mathbf{W}}^{T} \mathbf{h}(\mathbf{x}) \text {. }
$$

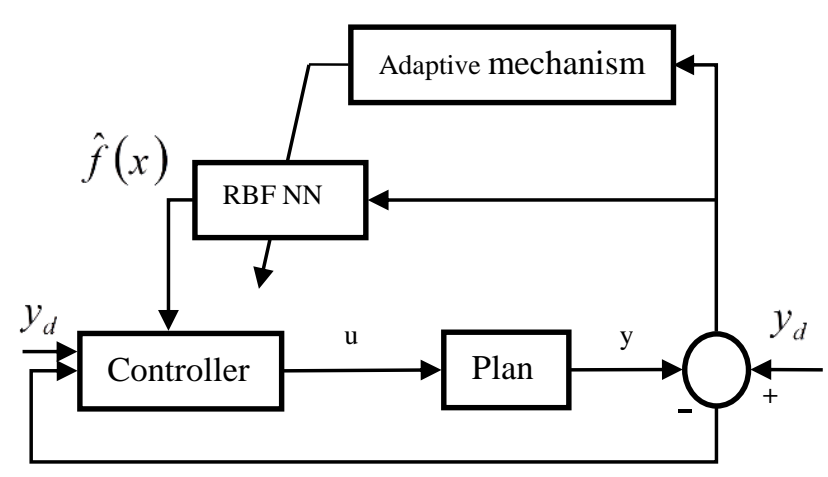

Figure 2. Block diagram of the control scheme.

\subsection{Sliding Mode Control}

sliding mode control, or SMC, is a nonlinear control method that alters the dynamics of a nonlinear system by application of a discontinuous control signal that forces the system to "slide" along a cross-section of the system's normal behavior. The control problem is to get the state $\mathbf{x}=\left[\begin{array}{llll}x & \dot{x} & \ldots & x^{(n-1)}\end{array}\right]$ to track a specific time-varying state $\mathbf{x}_{d}$ in the presence of model imprecision on $f(x)$ and $g(x)$. A time-varying surface $s(t)$ is defined in the state space $\mathfrak{R}^{n}$ by 
equating the variable $s(\mathbf{x}, t)$, defined below, to zero [15].

$$
s(\mathbf{x}, t)=\left(\frac{d}{d t}+\lambda\right)^{n-1} \tilde{\mathbf{x}}(t)
$$

and $\lambda$ is a strictly positive constant, taken to be the bandwidth of the system, and $\widetilde{\mathbf{x}}(t)=\mathbf{x}(t)-\mathbf{x}_{d}(t)$ is the error in the output state. The simplified, 1st-order problem of keeping the scalar $s$ at zero ( now be achieved by choosing the control law u of Eq. (11) such that outside of $s(t)$

$$
\frac{1}{2} \frac{d}{d t} s^{2} \leq-\eta|s|
$$

where $\eta$ is a strictly positive constant. take the control law as:

$$
u(t)=\hat{u}(t)-k(x, t) \operatorname{sgn}(s(t))
$$

By choosing $k(x, t)$ large enough such as $k(x, t)=F(x, t)+\eta$

\section{Implementation}

Consider dynamic equation of $\mathrm{AUV}$ and tracking error $e(t)=\eta_{d}(t)-\eta(t)$ and sliding mode function as $\mathbf{s}=\dot{\mathbf{e}}-\boldsymbol{\Lambda} \mathbf{e} \quad$ where $\quad \boldsymbol{\Lambda}=\boldsymbol{\Lambda}^{T}=\left[\begin{array}{llll}\lambda_{1} & \lambda_{2} & \ldots & \lambda_{n}\end{array}\right]^{T}>0$ such that $s^{n-1}+\lambda_{n-1} s^{n-2}+\ldots+\lambda_{1}$ is Hurwitz. According to sliding surface:

$$
\begin{aligned}
\mathbf{M} \dot{\mathbf{s}} & =\mathbf{M}\left(\ddot{\boldsymbol{\eta}}_{d}-\ddot{\boldsymbol{\eta}}+\boldsymbol{\Lambda} \dot{\mathbf{e}}\right) \\
& =\mathbf{M}\left(\ddot{\boldsymbol{\eta}}_{d}+\boldsymbol{\Lambda} \dot{\mathbf{e}}\right)-\mathbf{M}(\ddot{\boldsymbol{\eta}}) \\
& =\mathbf{M}\left(\ddot{\boldsymbol{\eta}}_{d}+\boldsymbol{\Lambda} \dot{\mathbf{e}}\right)+\mathbf{C} \dot{\boldsymbol{\eta}}+\mathbf{G}+\boldsymbol{\tau}_{\mathbf{d}}-\boldsymbol{\tau} \\
& =-\mathbf{C s}-\boldsymbol{\tau}+\mathbf{f}(x)+\boldsymbol{\tau}_{\mathbf{d}}
\end{aligned}
$$

where $\quad \mathbf{f}(x)=\mathbf{M} \ddot{\boldsymbol{\eta}}_{r}+\mathbf{C} \dot{\boldsymbol{\eta}}_{r}+\mathbf{G}, \quad \dot{\boldsymbol{\eta}}_{r}=\dot{\boldsymbol{\eta}}_{d}+\boldsymbol{\Lambda e} . \quad$ The goal is to design a stable robust controller without any modeling information. In this section, we use RBF to approximate $\mathbf{f}(x)$. Consequently, $\mathbf{f}-\hat{\mathbf{f}}=\tilde{\mathbf{W}}^{\mathrm{T}} \mathbf{h}+\varepsilon$, $\tilde{\mathbf{W}}=\mathbf{W}-\hat{\mathbf{W}}$, and $\|\mathbf{W}\|_{F} \leq \mathbf{W}_{\max }$. Eventually, the input of RBF should be chosen as $\mathbf{x}=\left[\begin{array}{lllll}\mathbf{e}^{T} & \dot{\mathbf{e}}^{T} & \boldsymbol{\eta}_{d}^{T} & \dot{\boldsymbol{\eta}}_{d}^{T} & \ddot{\boldsymbol{\eta}}_{d}^{T}\end{array}\right]$.

Control law

Control law for the dynamic equation of AUV Eq. (1) with robust term $\mathbf{v}=-\left(\varepsilon_{N}+b_{d}\right) \operatorname{sgn}(\mathbf{s})$ proposed as:

$$
\boldsymbol{\tau}=\hat{\mathbf{f}}(\mathbf{x})+\mathbf{K}_{\mathbf{v}} \mathbf{s}-\mathbf{v},
$$

The corresponding RBF adaptive law is designed as:

$$
\dot{\hat{\mathbf{W}}}=\boldsymbol{\Gamma} \mathbf{h} \mathbf{s}^{\mathbf{T}}, \quad \boldsymbol{\Gamma}=\boldsymbol{\Gamma}^{T}>0
$$

$\mathbf{M} \dot{\mathbf{r}}=-\left(\mathbf{K}_{\mathbf{v}}+\mathbf{C}\right) \mathbf{s}+\zeta_{1}$

where $\zeta_{1}=\tilde{\mathbf{W}}^{\mathbf{T}} \varphi+\left(\boldsymbol{\varepsilon}+\boldsymbol{\tau}_{\mathbf{d}}\right)+\mathbf{v}$

\subsection{Stability Analysis}

The closed system stability proof was shown with two steps as follows. Firstly, define Lyapunov function as

$$
\begin{aligned}
\mathbf{L} & =\frac{1}{2} \mathbf{s}^{\mathbf{T}} \mathbf{M} \mathbf{s}+\frac{1}{2} \operatorname{tr}\left(\tilde{\mathbf{W}}^{\mathbf{T}} \boldsymbol{\Gamma}^{-1} \tilde{\mathbf{W}}\right) \\
& \Rightarrow \quad \dot{\mathbf{L}}=\mathbf{s}^{\mathbf{T}} \mathbf{M} \dot{\mathbf{s}}+\frac{1}{2} \mathbf{s}^{\mathbf{T}} \dot{\mathbf{M}} \mathbf{s}+\operatorname{tr}\left(\tilde{\mathbf{W}}^{\mathbf{T}} \boldsymbol{\Gamma}^{-1} \dot{\tilde{\mathbf{W}}}\right)
\end{aligned}
$$

Secondly, inserting (17) into above yields

$$
\begin{aligned}
& \dot{\mathbf{L}}=-\mathbf{S}^{\mathbf{T}} \mathbf{K}_{\mathbf{v}} \mathbf{s}+\frac{1}{2} \mathbf{S}^{\mathbf{T}}(\dot{\mathbf{M}}-2 C) \mathbf{s} \\
& +\operatorname{tr} \tilde{\mathbf{W}}^{\mathrm{T}}\left(\boldsymbol{\Gamma}^{-\mathbf{1}} \dot{\tilde{\mathbf{W}}}+\mathbf{h} \mathbf{s}^{\mathbf{T}}\right)+\mathbf{s}^{\mathbf{T}}\left(\boldsymbol{\varepsilon}+\boldsymbol{\tau}_{\mathbf{d}}+\mathbf{v}\right)
\end{aligned}
$$

Since the skew-symmetric characteristics of AUV dynamic; a) $(\dot{\mathbf{M}}-2 C) \mathbf{s}=0$, b) $\operatorname{tr}\left(\tilde{\mathbf{W}}^{\mathbf{T}} \mathbf{h} \mathbf{s}^{\mathbf{T}}\right)=\mathbf{s}^{\mathbf{T}} \tilde{\mathbf{W}}^{\mathbf{T}} \mathbf{h}$, and c) $\quad \dot{\mathbf{L}}=-\mathbf{s}^{\mathbf{T}} \mathbf{K}_{\mathbf{v}} \mathbf{s}+\mathbf{s}^{\mathbf{T}}\left(\boldsymbol{\varepsilon}+\boldsymbol{\tau}_{\mathbf{d}}+\mathbf{v}\right) \quad$ so $\dot{\mathbf{L}}=-\mathbf{s}^{\mathbf{T}} \mathbf{K}_{\mathbf{v}} \mathbf{s}+\mathbf{s}^{\mathbf{T}}\left(\boldsymbol{\varepsilon}+\boldsymbol{\tau}_{\mathbf{d}}+\mathbf{v}\right)$ Moreover $\mathbf{s}^{\mathbf{T}}\left(\boldsymbol{\varepsilon}+\boldsymbol{\tau}_{\mathbf{d}}+\mathbf{v}\right)=\mathbf{s}^{\mathbf{T}}\left(\boldsymbol{\varepsilon}+\boldsymbol{\tau}_{\mathbf{d}}\right)-\|\mathbf{s}\|\left(\boldsymbol{\varepsilon}_{N}+b_{\mathbf{d}}\right) \leq 0$ eventually $\dot{\mathbf{L}} \leq-\mathbf{s}^{\mathbf{T}} \mathbf{K}_{\mathbf{v}} \mathbf{s} \leq 0$.

\section{Simulation Results}

\subsection{Steering}

The initial condition and reference circular planar trajectory are chosen as $\mathbf{x}(t)=\left[\begin{array}{llll}1.0 & 0 & 0.05 & 0.05\end{array}\right]^{T}$ and $x_{\text {des }}=10 \times \cos (0.2 t)+1, y_{\text {des }}=10 \times \sin (0.2 t)$, respectively. In addition, underwater ocean current modeled as disturbance

$u_{d i s}=\mathbf{v}_{C} \cos \beta$

$v_{\text {dis }}=\mathbf{v}_{C} \sin \beta$

where $\mathbf{v}_{C}=\sqrt{u^{2}+v^{2}}$ is current speed and $\beta$ is angle between the heading and the direction of the wave (rad). The time of simulation is set at 100 seconds meanwhile the needed time for one circle is 85 seconds. Number of the hidden node is 7 and we chose control law parameters given as $b=1$, $\lambda=2 I_{2 \times 2}, \mathbf{K}_{v}=100 I_{2 \times 2}, \varepsilon_{N}=0.75, b_{d}=0.25$, and $c=0.05\left[\begin{array}{lllllll}-1.5 & -1 & -0.5 & 0 & 0.5 & 1 & 1.5 \\ -1.5 & -1 & -0.5 & 0 & 0.5 & 1 & 1.5\end{array}\right]$

Other constants of the AUV are shown in [14]. the 2D path is presented in Figure 3. States that represent position and velocity of the AUV are expressed in Figure 4 and Figure 5. The estimation of $\mathbf{f}(x)$ is shown in Figure 6.The control signals are illustrated in Figure 7. 
Although precision tracking can be achievable through greater sliding gain robust term, greater sliding gain leads to high control effort which cannot implement in practical test. Furthermore, we can reach accurate estimation of $\mathbf{f}(x)$ with increasing of $b$ but it enhances tracking error also tracking error reduces with decreasing of $b$.

\subsection{Diving}

The initial condition is $\mathbf{x}(t)=\left[\begin{array}{llll}0.6 & 0.3 & 0.5 & 0.5\end{array}\right]^{T}$. Number of the hidden node is 5 and we chose control law parameters given as $b=3, \lambda=2 I_{2 \times 2}$, $\mathbf{K}_{v}=100 I_{2 \times 2}, \varepsilon_{N}=0.5, b_{d}=0.1$, and $c=0.05\left[\begin{array}{lllll}-1 & -0.5 & 0 & 0.5 & 1 \\ -1 & -0.5 & 0 & 0.5 & 1\end{array}\right]$

States that represent position and velocity of the AUV are expressed in Figure 8 and Figure 9. The estimation of $\mathbf{f}(x)$ is shown in Figure 10.The control signals are illustrated in Figure 11.

As depicted in Figures, on the contrary of optimal control method such as SDRE, proposed control method regulate to desired depth because of sliding mode robust augmented term and guarantee appropriate trajectory tracking due to hybrid disturbance rejection mechanism. The simulations illustrate the positive aspects of the approach, as well as some of the potential drawbacks which can be compensated by carefully choosing control gains and parameters.

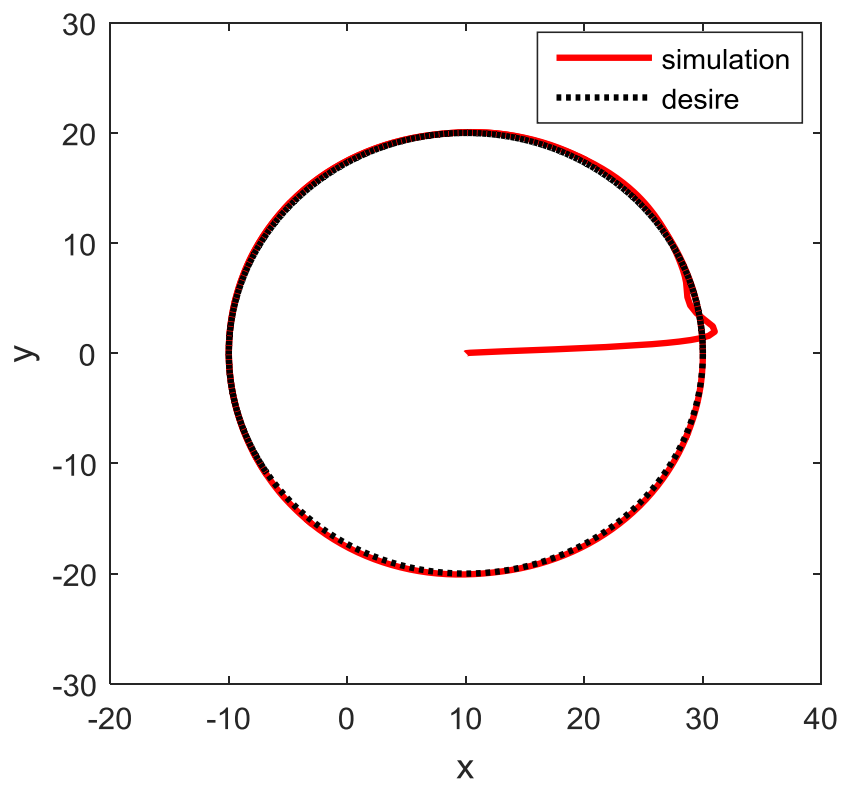

Figure 3. 2D steering generated path of REMUS model.
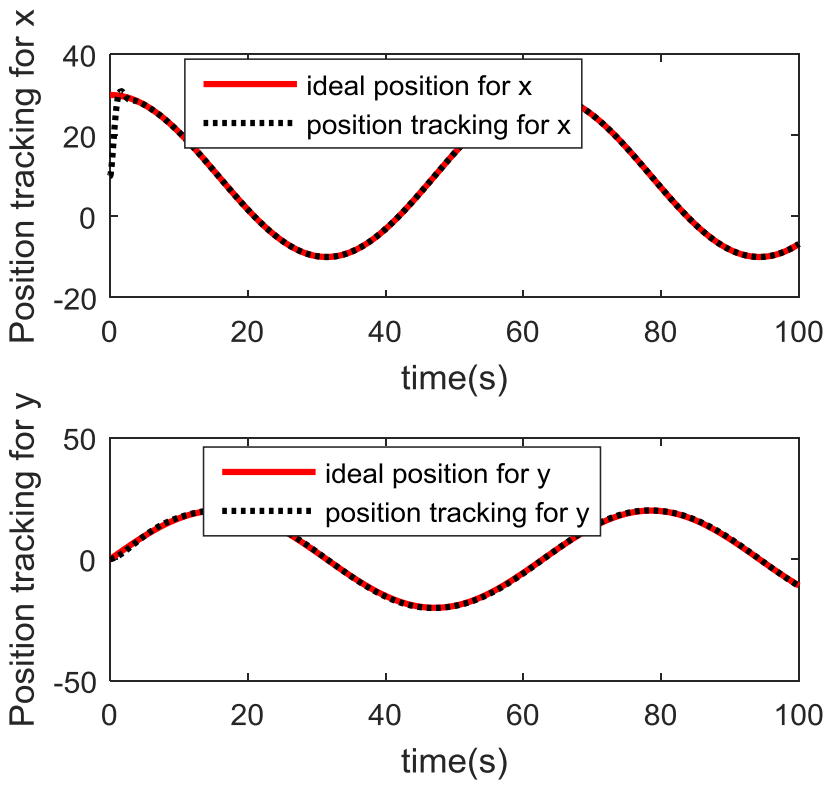

Figure 4. states of the position of REMUS.
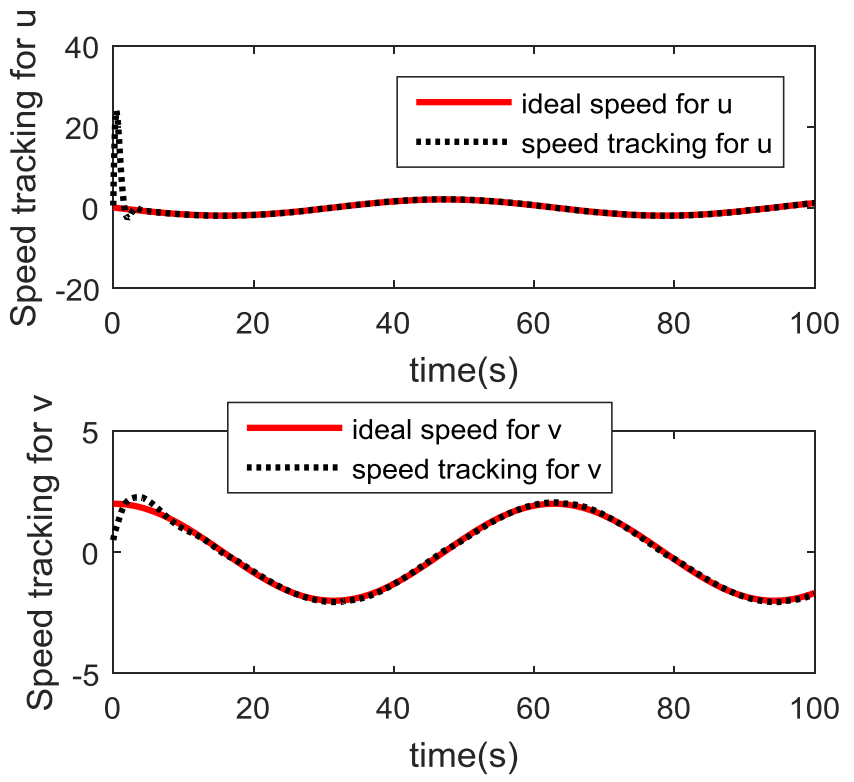

Figure 5. linear velocities of REMUS.

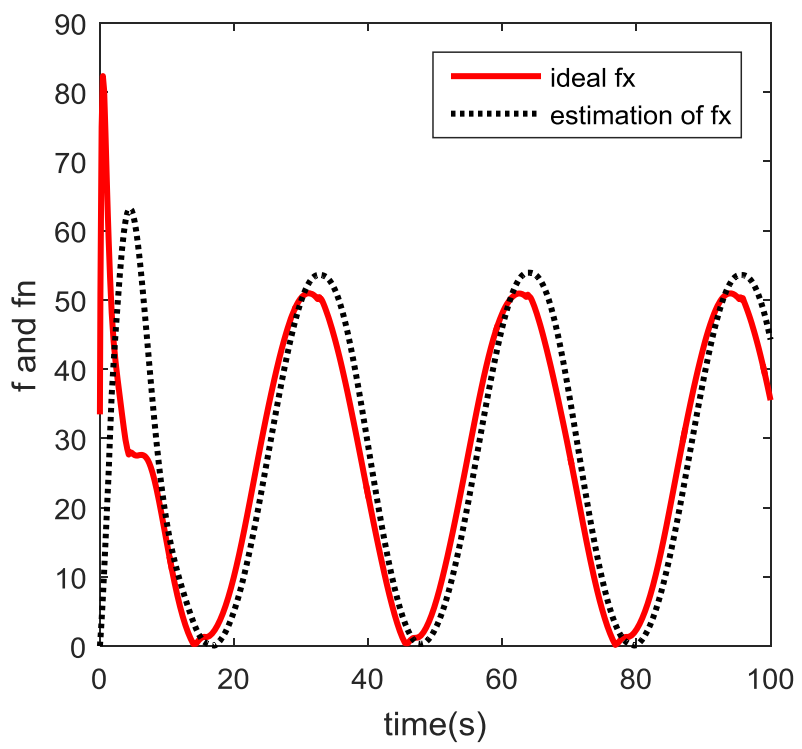

Figure 6. Estimation of $f(x)$. 

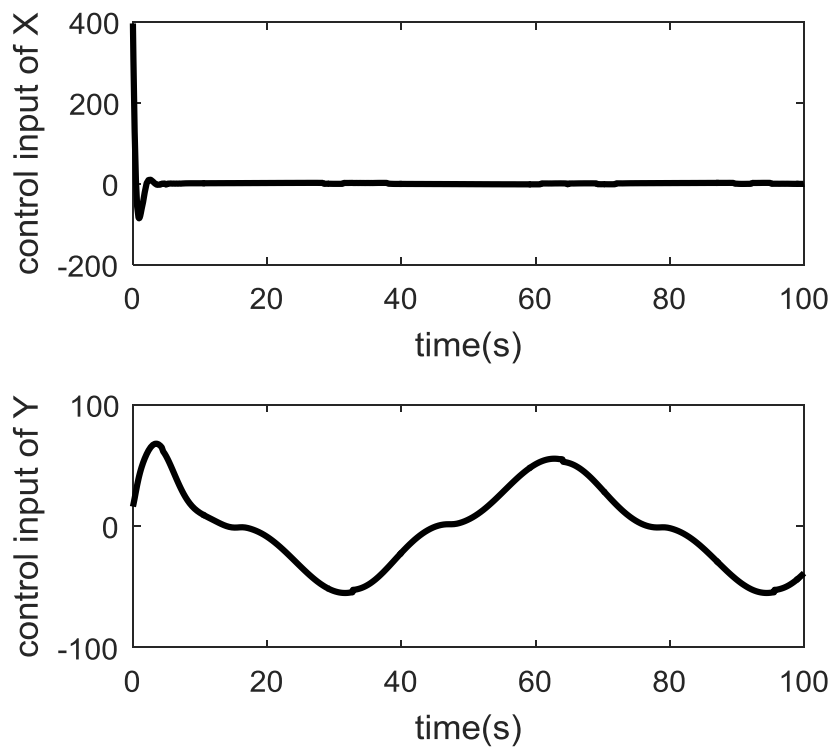

Figure 7. control signals.
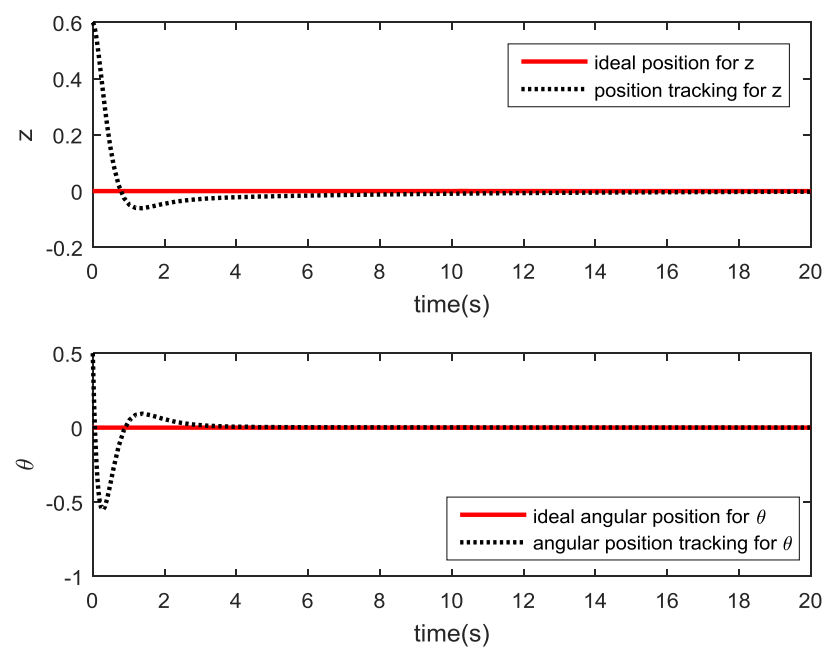

Figure 8. Diving states of REMUS
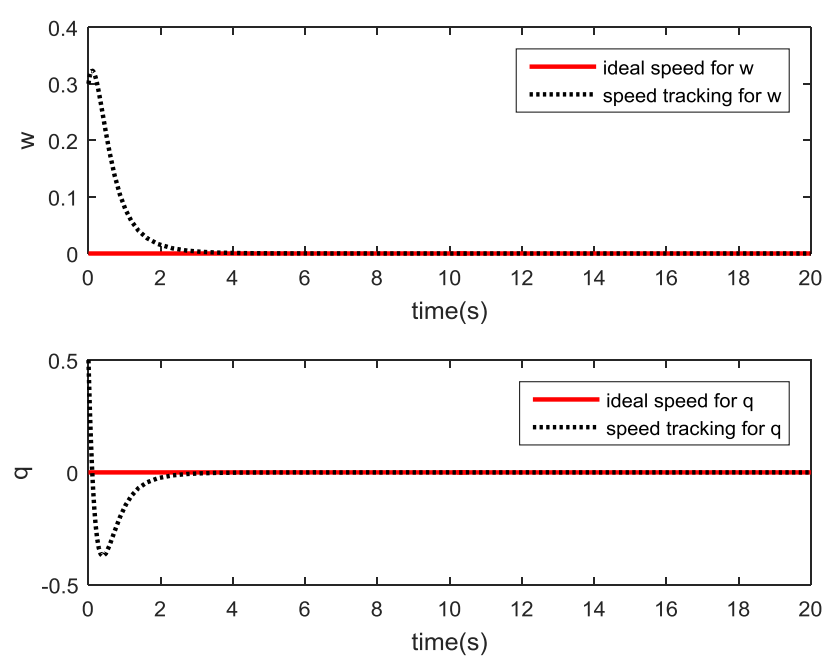

Figure 9. Linear and angular velocities of REMUS in diving mode

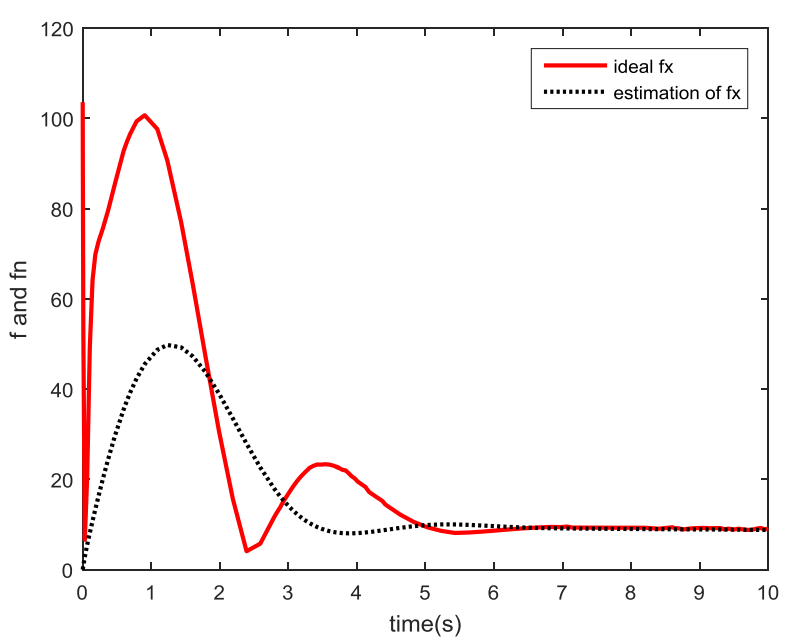

Figure 10. Estimation of $f(x)$ in diving mode
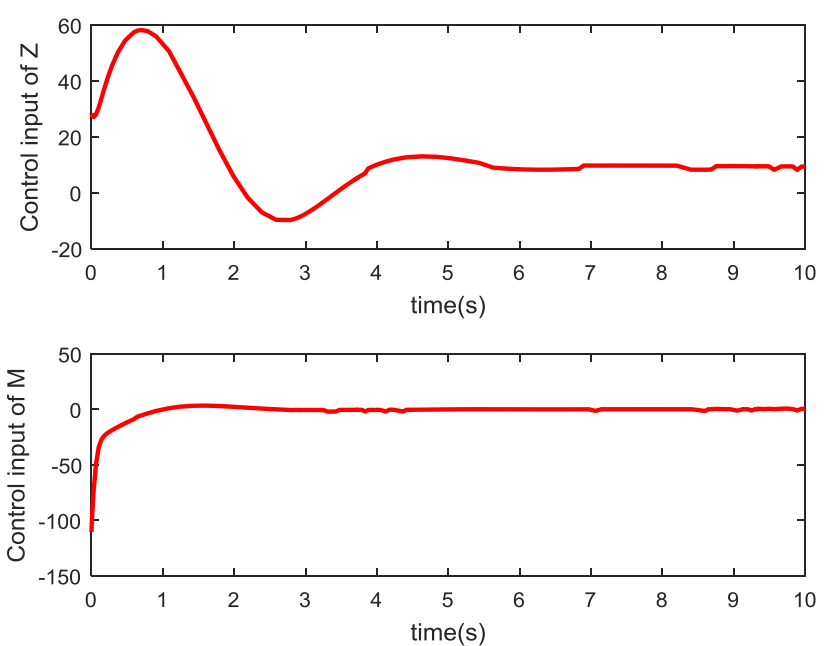

Figure 11. Control efforts in diving mode

\section{Conclusions}

In this work presents a generic approach for designing of nonlinear control via the adaptive radial based function with robust term. REMUS was selected for implementing the control law. Adaptive radial basis function neural network with sliding mode robust term is applied for several reasons such as; reaching to exact dynamic model of AUVs is not possible, a mathematic model of AUV includes high nonlinearities and complex behavior, and AUVs work in unknown environment so can be affected by circumstances. Consequently, adaptive RBF NN is used to estimate $\mathrm{f}(\mathrm{x})$ and sliding mode robust term is used to overcome uncertainty in AUV modeling. The simulation results show that this method can control the steering and diving autopilot system in point-topoint motion (regulations). The last but not the least, stability proof of suggested control scheme is demonstrated for autonomous underwater vehicles by Lyapunov theory.

This contribution provides an extension to the current 
results, but is restricted to the types of trajectories it can

track especially complex 3D trajectory which deserves attention. Another research option is to account for fully coupled dynamic model of 6-DOF AUVs while designing the proposed control method then have comparative study between trajectory tracking error and energy consumption of decoupled dynamics and coupled dynamics.

\section{Acknowledgement}

I would like to thank anonymous referees for some valuable suggestions, which have resulted in the improvement of this work. This work is supported by Young Researchers and Elite Club, BuinZahra Branch, Islamic Azad University, BuinZahra, Iran.

\section{References}

1- Chu, Z. and D. Zhu. (2016), adaptive sliding mode heading control for autonomous underwater vehicle including actuator dynamics. in OCEANS 2016Shanghai. 2016. IEEE.

2- Geranmehr, B. and S.R. Nekoo, (2015), nonlinear suboptimal control of fully coupled non-affine sixDOF autonomous underwater vehicle using the statedependent Riccati equation. Ocean Engineering, 2015. 96: p. 248-257.

3- Geranmehr, B. and S.R. Nekoo. (2014), the nonlinear suboptimal diving control of an autonomous underwater vehicle. in Robotics and Mechatronics (ICRoM), 2014 Second RSI/ISM International Conference on. 2014. IEEE.

4- Geranmehr, B. and S.R. Nekoo. (2014) the statedependent set-point regulation and tracking control of horizontal motion of $A U V$. in Robotics and Mechatronics (ICRoM), Second RSI/ISM International Conference on. 2014. IEEE.

5- Rezazadegan, F. and K. Shojaei. (2013) an Adaptive Control Scheme for 6-DOF Control of an AUV Using Saturation Functions. in Proceedings of the 3rd International Conference on Intelligent Computational Systems, April.

6- Mazinan, A., (2016) High-performance robust three-axis finite-time attitude control approach incorporating quaternion based estimation scheme to overactuated spacecraft. International Journal of Engineering-Transactions A: Basics, 2016. 29(1): p. 53.

7- Lewis, F.L., K. Liu, and A. Yesildirek, (1995) Neural net robot controller with guaranteed tracking performance. IEEE Transactions on Neural Networks, 1995. 6(3): p. 703-715.

8- Daachi, M., et al., (2015) A radial basis function neural network adaptive controller to drive a powered lower limb knee joint orthosis. Applied Soft Computing, 2015. 34: p. 324-336.

9- Lei, X. and P. Lu, (2014) The adaptive radial basis function neural network for small rotary-wing unmanned aircraft. IEEE Transactions on Industrial Electronics, 2014. 61(9): p. 4808-4815.

10- Fateh, M.M., S.M. Ahmadi, and S. Khorashadizadeh, (2014) adaptive RBF network control for robot manipulators. Journal of $\mathrm{AI}$ and Data Mining, 2014. 2(2): p. 159-166.

11- Chu, Y. and J. Fei, (2015) adaptive global sliding mode control for MEMS gyroscope using RBF neural network. Mathematical Problems in Engineering, 2015.

12- Fossen, T.I., (1994) Guidance and control of ocean vehicles. John Wiley \& Sons Inc.

13- SNAM, E., (1952) nomenclature for treating the motion of a submerged body through a fluid JR. New York: Technical and Research Bulletin, 1952: p. 1-5. 14- Prestero, T., (2001) verification of a six-degree of freedom simulation model for the REMUS autonomous underwater vehicle. Massachusetts Institute of Technology and Woods Hole Oceanographic Institution.

15- Slotine, J.-J.E. and W. Li, (1991) applied nonlinear control. Vol. 199. prentice-Hall Englewood Cliffs, NJ. 